\title{
Pharmacokinetics and pharmacodynamics
}

\section{F Rimmington}

Department of Anaesthesia, Charlotte Maxeke Johannesburg Academic Hospital, University of the Witwatersrand, South Africa Corresponding author, email: farrahgermanus@gmail.com

\section{Summary}

Pharmacokinetics and pharmacodynamics are vast and complex topics with medical textbooks dedicating entire chapters to explain the details. Due to this, only some of the many relevant concepts have been explained. Compartment models have not been discussed despite their importance to the anaesthetist.

These two concepts have been described as mathematical expressions of the relationship between drug concentration and time for pharmacokinetics, and drug concentration and effect for pharmacodynamics. Concentration provides the link between pharmacokinetics and pharmacodynamics and will enable rational dosing. Pharmacokinetics consists of four linked processes namely absorption, distribution, biotransformation and excretion. Clinical pharmacokinetics including bioavailability, bioequivalence, first-order and zero-order kinetics as well as context-sensitive half-life have been discussed. A basic explanation of drug-receptor interactions, dose-response relationships and therapeutic index is given for pharmacodynamics.

The purpose of understanding these concepts is to enable doctors to simultaneously achieve the desired therapeutic effects while minimising adverse effects. This is particularly relevant to the practising anaesthetist where multiple agents are required to produce optimal anaesthesia.

Keywords: pharmacokinetics, pharmacodynamics

\section{Introduction}

Pharmacokinetics is often simplistically explained in medical textbooks as 'what the body does to the drug' and pharmacodynamics as 'what the drug does to the body.' These two concepts have also been explained as mathematical expressions of the relationship between drug concentration and time for pharmacokinetics, and drug concentration and effect for pharmacodynamics. ${ }^{2}$ These relationships are illustrated in Figure 1. Concentration provides the link between pharmacokinetics and pharmacodynamics and will enable rational dosing. ${ }^{2}$ The aim of understanding these concepts is to assist doctors administering drugs to achieve the desired therapeutic effects while minimising adverse effects. ${ }^{2}$

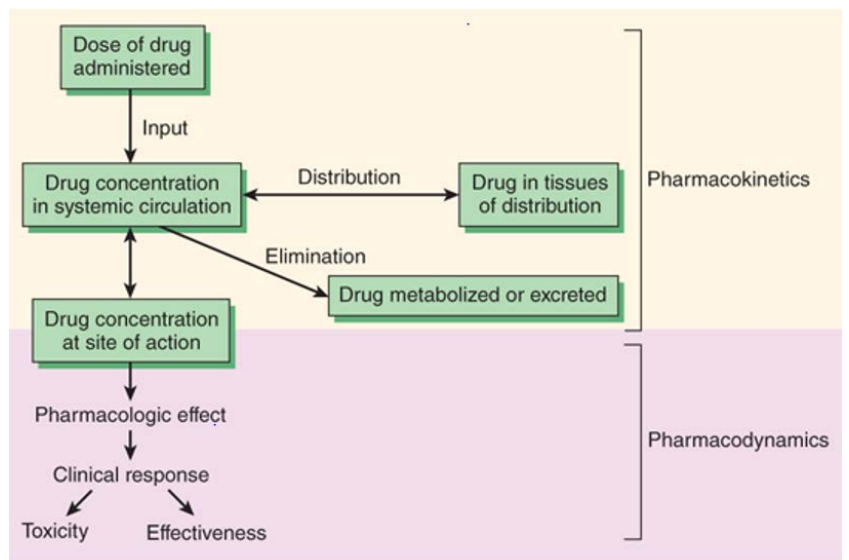

Figure 1: Relationship between pharmacokinetic and pharmacodynamic components ${ }^{3}$

\section{Discussion}

\section{Pharmacokinetic principles}

Pharmacokinetics consists of four linked processes including absorption, distribution, biotransformation and excretion. ${ }^{4}$

Absorption involves drug movement from the site of administration to the site of measurement. ${ }^{1} \mathrm{~A}$ drug must pass through the cellular plasma membrane to exert its effect. This is done via a transport process. This may be a passive process such as simple diffusion or an active one that requires energy and is carrier mediated. ${ }^{1}$ Multiple factors can affect drug absorption including blood flow and the physical and chemical properties of the drug. Non-ionised drugs are lipid soluble and can diffuse across cell membranes while ionised forms are lipid insoluble and unable to dissolve in the lipid membrane. Degree of ionisation depends on the pKa of the drug and the $\mathrm{pH}$ of the environment. The pKa of a drug is the $\mathrm{pH}$ value of the solution in which $50 \%$ of the drug is dissociated, therefore half the drug is ionised and half non-ionised. ${ }^{1}$ If the $\mathrm{pH}$ is inappropriate, the drug cannot become unionised and will be ineffective as demonstrated when lignocaine is injected into the acidic environment of infected tissues. ${ }^{3}$

Multiple routes of drug administration exist, each with their own advantages and disadvantages. Absorption from the gastrointestinal tract occurs with orally administered drugs. ${ }^{1}$ This exposes the drug to gastric $\mathrm{pH}$, enzymes and first-pass hepatic metabolism. ${ }^{4}$ Most drugs are absorbed from the small intestine 
instead of the stomach due to the large absorptive area and longer transit time. ${ }^{4}$ Sublingual and buccal drug absorption bypasses the liver and first-pass metabolism, as venous drainage from the mouth and oesophagus flows into the superior vena cava and not the portal system. ${ }^{4}$ Rectal drug administration may be useful in the paediatric or unconscious patient with no intravenous access, however absorption can be erratic and unpredictable. ${ }^{1}$ The lower rectum drains directly into the inferior vena cava via the middle and inferior rectal veins, bypassing the portal system. The upper rectum drains into the portal system via the superior rectal vein and undergoes first-pass metabolism. ${ }^{1}$

Absorption for subcutaneous and intramuscular injection occurs along diffusion gradients from the site injected to the plasma. ${ }^{1}$ The rate of diffusion depends on regional blood flow and solubility of drug. ${ }^{1}$ Absorption is completely bypassed by intravenous injections as the total dose goes into the bloodstream. ${ }^{4}$

Distribution occurs after absorption and involves the reversible transfer of a drug from one location in the body to another.' It can be rate limited by perfusion or permeability. ${ }^{1}$ Drugs will initially distribute rapidly to well perfused organs such as the brain, liver and kidney then slowly to less perfused organs like viscera, skin and muscle. ${ }^{1}$ Pharmacokinetic models use these differing rates of distribution to formulate compartment models. Drug distribution is dependent on the same principles as absorption, namely degree of ionisation, fat solubility, size of the molecules and protein-binding. ${ }^{1}$ Drug molecules obey the law of mass action. ${ }^{4}$ Once the plasma concentration of the drug is greater than the tissue concentration, the drug will move from the plasma to the tissue. ${ }^{4}$ Equilibration occurs when the concentration in the tissue and plasma is the same. Following this, redistribution occurs as the drug moves from the tissue back to the blood when the concentration in the plasma is less than the concentration in the tissue. ${ }^{4}$

Volume of distribution ( $\mathrm{Vd}$ ) is the apparent volume that a dose of drug has distributed into. ${ }^{1,4}$ It does not represent a real volume but the volume into which a drug dose would need to distribute, in order to produce the observed plasma concentration. ${ }^{4}$ The equation for $\mathrm{Vd}$ is as follows:

$\mathrm{Vd}=\frac{\text { total amount of drug in body/dose }}{\text { concentration of drug in plasma }}$

Multiple factors can affect the Vd. Movement of a drug out of the vascular compartment is determined by drug characteristics, such as protein-binding, pKa, lipid solubility, molecular size, degree of binding to other tissues and patient characteristics such as age and disease state. ' If a significant amount of drug leaves the vascular compartment, it will have a large Vd. Highly plasma protein bound drugs cannot move freely so stay in the vascular space and will have a small Vd. Albumin binds many acidic drugs while $a_{1}$-acid glycoprotein binds basic drugs. ${ }^{4}$ Low albumin reduces binding causing an increase in free drug. Pregnant patients have decreased total plasma proteins, so the dose of a highly protein bound drug such as propofol will have to be reduced. ${ }^{1}$ Drugs with significant tissue binding to cellular components will result in a low plasma concentration and a large Vd. Basic drugs tend to be absorbed more easily by most tissues and hence have a large $\mathrm{Vd}$ while the opposite is true for acidic drugs. ${ }^{1}$

Biotransformation is the chemical process by which drug molecules are altered in the body. ${ }^{4}$ The primary organ of metabolism is the liver, except for esters which are hydrolysed in the plasma or tissues. ${ }^{4}$ The end products are usually watersoluble to allow for renal excretion. ${ }^{4}$ This usually occurs in two sequential phases. Phase 1 is metabolic transformation by oxidation, reduction or hydrolysis via the cytochrome P450 enzymes which makes the drug more water soluble., ${ }^{1,4}$ Phase 2 is conjugation reactions to create inactive conjugates which can be eliminated in urine or faeces. ${ }^{1,4}$ Notably, phase 1 metabolites can be excreted without phase 2 reactions occurring and a phase 2 reaction does not always require a phase 1 reaction to precede it. $^{4}$

The cytochrome P450 enzyme system has 30 different CYP enzymes involved in drug metabolism. ${ }^{1}$ These enzymes are largely responsible for the variable metabolism of drugs in different people due to genetic variability. ${ }^{1}$ It is located on the endoplasmic reticulum membrane mainly in the liver but also in the kidney and gut wall. They are easily induced or inhibited by drugs.' Inhibition of these enzymes will result in reduced breakdown of a drug while induction will increase breakdown and therefore decrease the plasma drug concentration. ${ }^{4}$

Most anaesthetic drugs are metabolised in the liver. ${ }^{1,4}$ The rate of drug metabolism depends on how much drug is delivered to the liver via hepatic blood flow and enzyme activity in the liver to metabolise the drug. ${ }^{1}$ Orally administered drugs enter the liver via the portal vein where rapid biotransformation occurs and this results in less active drug available for distribution. Conversely intravenous drugs do not undergo this first-pass effect. ${ }^{1}$

For most drugs, only a fraction that enters the liver will be metabolised and this is called the extraction ratio. ${ }^{4}$

Drugs with a high extraction ratio are flow limited or perfusion dependent, with liver blood flow determining the rate of clearance. These drugs follow first-order kinetics and the rate of metabolism is rapid, e.g. propofol and sufentanil. ${ }^{1}$

Drugs with a low hepatic extraction ratio are capacity limited as clearance depends on enzyme activity. If liver enzymes are induced, then clearance will increase, but if the liver is damaged or diseased, then clearance will decrease. These drugs follow zero-order kinetics occurs with a slow rate of metabolism, e.g. alfentanil and phenytoin. ${ }^{1}$

Many drugs and their metabolites are excreted via the kidneys. ${ }^{2,4}$ Excretion will depend on glomerular filtration rate (GFR), active tubular secretion, passive tubular absorption and renal disease. ${ }^{1}$ Some drugs undergo enterohepatic recirculation where they are excreted in bile, then released into the small intestine where 
the drug is reabsorbed into the circulation and subsequently returned to the liver. ${ }^{4}$

Other pathways include non-organ clearance such as Hofmann elimination and hydrolysis by plasma esterases. ${ }^{1}$

\section{Clinical pharmacokinetics}

\section{Bioavailability}

This is the fraction or percentage of unchanged drug that reaches the systemic circulation following administration via any route. It will differ depending on the route of administration. An intravenous drug will have a bioavailability of one or $100 \%$. Any other route will be less than that due to incomplete absorption and metabolism before reaching the systemic circulation. ${ }^{1}$ This is illustrated in Figure 2.

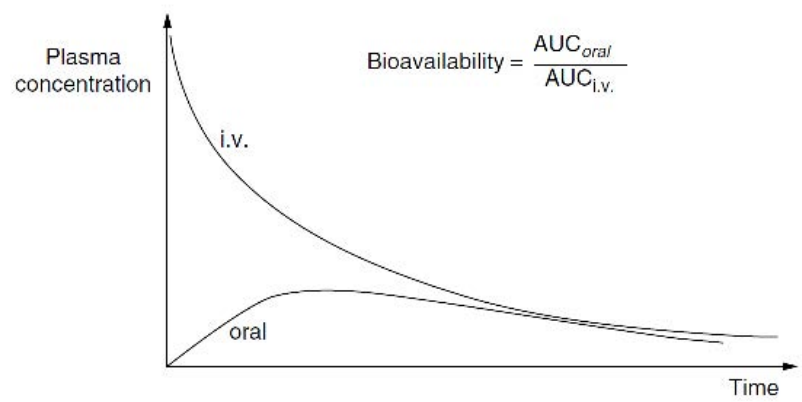

Figure 2: Bioavailability may be estimated by comparing area under the curves $^{5}$

\section{Bioequivalence}

This is used to compare the active ingredient in a generic drug with the approved original drug formulation. Assessment is done by comparing the bioavailability of the two drugs. ${ }^{1}$ There are criteria required to be considered equivalent. The generic firstly must have an area under the curve (AUC) of $80-125 \%$ of the original drug, secondly a maximum concentration $\left(C_{\max }\right)$ of more than $90 \%$ and thirdly a similar time to maximum concentration $\left(T_{\max }\right)$ when given at the same molar dose. ' If these criteria are met, it implies that the generic drug releases its active ingredient into plasma at about the same rate as the original drug. ${ }^{1}$

\section{First-order kinetics (linear kinetics)}

In linear kinetics, clearance is in proportion to the amount of drug present in the plasma. The more drug present, the faster the elimination. After a bolus, the half-life is constant while the rate of metabolism slows in proportion to the concentration. ${ }^{1}$ The rate limiting factor is the initial concentration.

\section{Zero-order kinetics (non-linear kinetics)}

This occurs with clearance of a constant amount of drug from plasma per unit time. Metabolism of the drug is constant while half-life decreases over time. If a large dose is given, then enzyme systems will become saturated. A commonly quoted example which follows this model is ethanol.

Importantly, large doses of a drug can overwhelm the rate of metabolism and change metabolism from first-order to zeroorder kinetics.

Half-life $\left(t_{1 / 2}\right)$

This is the time it takes for $50 \%$ of the drug to be eliminated from the body. ${ }^{1}$ It is assumed that drugs are removed from the body after five half-lives.

$t_{1 / 2}=\frac{0.693 \times V d}{\text { clearance }}$

(0.693 $=\mathrm{k}=$ elimination constant $)$

Half-life is directly proportional to volume of distribution and inversely proportional to clearance.

\section{Context-sensitive half-time (CSHT)}

This is the time required for a $50 \%$ decrease in plasma drug concentration to occur after discontinuing a steady state infusion. ${ }^{4}$ Since it describes drug concentration in the plasma and not the effect site, it does not directly describe time to recovery. ${ }^{1}$ It attempts to explain the accumulation and distribution following different infusion durations. The CSHT will increase as the duration of the infusion increases. The context in CSHT refers to the duration of the infusion. ${ }^{4}$ Remifentanil is an exception as it has a constant CSHT regardless of the duration of the infusion. ${ }^{1}$ CSHT of various drugs are illustrated in Figure 3.

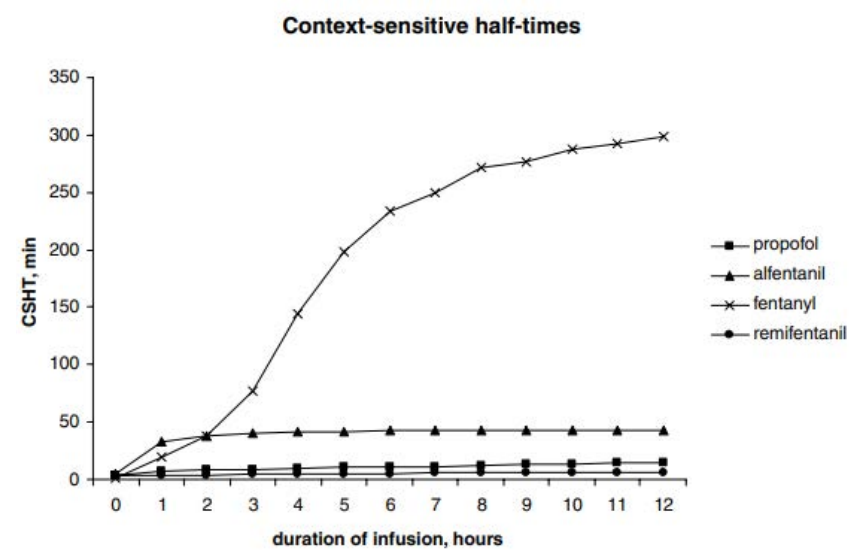

Figure 3: Variation in context-sensitive half-times with duration of infusion $^{6}$

\section{Infusions}

Steady state-plasma concentration (Css) is reached when the rate of administration is equal to the rate of elimination. ${ }^{1}$ If a drug that obeys first-order kinetics is given via multiple doses at fixed intervals, the time to reach Css would be $4-5$ half-lives. ${ }^{1}$ To overcome this, a loading dose is given to attain Css. To maintain a steady state the rate of administration must equal the rate of elimination. ${ }^{1}$ Accumulation can occur in an infusion when the 
rate of administration of the drug is greater than the elimination. This will depend on the drug's half-life and the dosing interval. Greater accumulation occurs with drugs with long half-lives and short dosing intervals.

\section{Pharmacodynamics}

This is explained as the mathematical expression of the relationship between drug concentration and effect. It incorporates concepts such as the binding of drugs to cells, drug uptake and intracellular drug metabolism. The biophase is the site where the drug exerts its action. Most drugs must leave the plasma to reach different tissues to exert their effect. ${ }^{5}$ This can cause a delay between time taken to reach a plasma concentration and the onset of clinical effect known as 'effect hysteresis.'

\section{Drug-receptor interactions}

The drug will need to bind to a receptor, form a drug-receptor complex to cause an effect. ${ }^{7}$ Receptors are proteins or polypeptides that have a binding site for the drug/ligand. The receptor may be on the cell surface or intra-cellularly. ${ }^{7}$ Only ligands that fit precisely bind to receptors. Receptors have three important properties. Specificity indicates that a specific receptor may only bind to a narrow range of ligands.? Affinity is the avidity with which receptors bind drugs and intrinsic activity or efficacy is the capacity to elicit a pharmacological response.?

\section{Dose response relationships}

A full agonist can elicit a maximal response following receptor occupation and activation while a partial agonist is unable to elicit the maximal response. ${ }^{7}$ An inverse agonist binds to the same receptor binding site as an agonist but exerts an opposite pharmacological effect as the agonist.' Competitive antagonists will bind in a reversible way to the receptor and inhibit the agonist effects by competing for receptor occupancy. Irreversible antagonists prevent the agonist from reaching maximal response and cannot be overcome by increasing the concentration of the agonist as the antagonist effect is not reversible.?

\section{Therapeutic ratio/index}

$E D_{50}$ is the effective dose that will produce a specified effect in $50 \%$ of individuals in whom it is studied while the $L_{50}$ is the lethal dose required to cause death in $50 \%$ of individuals. ${ }^{7} \mathrm{TD}_{50}$ is the toxic dose causing a specified toxic effect in $50 \%$ of individuals. The therapeutic ratio/index is the ratio between $\mathrm{LD}_{50}$ and $E D_{50}$ or $\mathrm{TD}_{50}$ and $\mathrm{ED}_{50} .{ }^{5}$ The higher the ratio, the safer the drug.

\section{Conclusion}

Understanding pharmacokinetics and pharmacodynamics will assist clinicians in selecting the correct drugs, doses and dosing intervals in different patients. This is particularly relevant to the practising anaesthetist where multiple agents are required to produce optimal anaesthesia. This topic can be considered the science while years of experience in titrating anaesthetic drugs can be thought of as the art.

\section{Conflict of interest}

The author declares no conflict of interest.

\section{Funding source}

None.

\section{ORCID}

F Rimmington (iD https://orcid.org/0000-0002-6086-8077

References

1. Meyer BH, Welch EH, Milner A, Möhr D. Applied pharmocokinetics. In: Milner A, Welch E, editors. Applied pharmacology in anaesthesiology and critical care. 1st ed. Medpharm Publications (Pty) Ltd; 2012. p. 1-32.

2. Nicholas $\mathrm{HG}$, Holford MB. Pharmacokinetics and pharmacodynamics: rational dosing and the time course of drug action. In: Katzung BG, editor. Basic \& clinical pharmacology. 8th ed. McGraw-Hill; 2001. p. 35-50.

3. Nicholas HG, Holford MB. Pharmacokinetics and pharmacodynamics: rational dosing and the time course of drug action. In: Katzung BG, editor. Basic \& clinical pharmacology. 8th ed: McGraw-Hill; 2001. p. 35-50. Figure 1-3, the relationship between pharmacokinetic and pharmacodynamic components; p. 36.

4. Morgan \& Mikhail's clinical anaesthesiology. 5th ed. McGraw-Hill Companies, Inc; 2013. chap 7. p. 143-53.

5. Peck T, Hill S, Williams M. Pharmacology for anaesthesia and intensive care 3rd ed. Cambridge University Press; 2008. Figure 2.1, bioavailability may be estimated by comparing area under the curves; $p .9$.

6. Peck T, Hill S, Williams M. Pharmacology for anaesthesia and intensive care. 3rd ed. Cambridge University Press; 2008. Figure 6.14, variation of context-sensitive half-time with duration of infusion; p. 78.

7. Etoe S. Pharmacodynamics. In: Milner A, Welch E, editors. Applied pharmacology in anaesthesia and critical care. 1st ed. Medpharm Publications (Pty) Ltd; 2012. p. 33-54. 